\title{
Improving the -3dB bandwidth of medium power GaN-based LEDs through periodic micro via-holes for visible light communications
}

Zheng Zhou ${ }^{1}$, Bing Yan ${ }^{1}$, DongdongTeng ${ }^{2}$, Lilin Liu ${ }^{1}$, Gang Wang ${ }^{1}$ *

${ }^{1}$ School of Electronics and Information Technology, ${ }^{2}$ School of Physics, Sun Yat-Sen University, Guangzhou, China 510275.

*Corresponding authors: liullin@mail.sysu.edu.cn; stswangg@mail.sysu.edu.cn

Abstract - Medium power GaN-based light emitting diode (LED) chips with periodic micro via-holes are designed and fabricated. The active area of each chip is $200 \mu \mathrm{m} \times 800 \mu \mathrm{m}$ and the diameter of each micro via-hole is $50 \mu \mathrm{m}$. For comparison, an LED chip with only one big via-hole (Diameter $=86.6 \mu \mathrm{m})$ is also fabricated under the same conditions as the control partner. Both kinds of LED chips have an equal effective PN junction area. Experimentally, the LED with periodic via-holes exhibits higher output optical power and the $-3 \mathrm{~dB}$ modulation bandwidth by about $33 \%$ and $48 \%$, respectively, than the LED with only one bigger via-hole. The method of concurrently improving modulation and optical performances of power-type LED chips through periodic micro via-holes take the advantages of easy fabrication, suitable for mass-production.

Keywords: LED with periodic micro via-holes, blue LED, Visible-light Communication (VLC), -3dB modulation bandwidth. 


\section{Introduction}

Gallium Nitride $(\mathrm{GaN})$ based light emitting diodes (LEDs) have been considered as the next generation lighting source due to its high efficiency, long lifetime, and high brightness. Thanks to the rapid success of commercial white LEDs, visible light communication (VLC) based on LEDs is expected to be a strong competitor of next generation high speed wireless communications. VLC has many advantages: a license-free of THz bandwidth, no electromagnetic interference, good safety, etc. [1]. However, the commercially available medium and high power LEDs (e.g. OSRAM ${ }^{\mathrm{TM}}$ ) only have very narrow bandwidth, about several $\mathrm{MHz}$, hindering the development of high speed VLC systems.

Significant research efforts are being directed towards the development of high speed power-type LEDs. Many methods, such as blue filtering, equalization (e.g. pre-equalization [2-3] and post-equalization [4-5]), and other special methods, had been proposed to improve the $-3 \mathrm{~dB}$ modulation bandwidth. H. L. Li et al. showed the phosphor-based white LED had $\sim 132 \mathrm{MHz}-3 \mathrm{~dB}$ bandwidth with optimized analog equalizers [6]. Tian P et al. designed GaN-based 10×10 micro-pixelated LED ( $\mu$ LED) arrays and the $\mu$ LED with a diameter of $45 \mu \mathrm{m}$ demonstrated a high electrical-to-optical modulation bandwidth of up to $270 \mathrm{MHz}$ [7]. Such $\mu$ LED arrays exhibited great potentials for high speed optical interconnects. D. H. Kwon et al. proposed an equalization design with pre-equalization and blue filtering, which extended the $-3 \mathrm{~dB}$ modulation bandwidth to $\sim 180 \mathrm{MHz}$ [8]. C. H. Yeh et al. demonstrated an optimal analogue front end circuit to increase the modulation bandwidth of phosphor-LED. Without using blue filter and post equalization, $\sim 32 \mathrm{MHz}$ modulation bandwidth was achieved [9]. Y. C. Lee et al. designed a white light LED driver which combined with the pre-equalizer technology. The optical modulation bandwidth could be increased to $10.9 \mathrm{MHz}[10]$. However, these methods had characteristics of high-cost, complex, low-yield, etc. Improving the $-3 \mathrm{~dB}$ bandwidth at the LED chip level is desired. 
For power-type LEDs, the poor current spreading and the resulted severe current-crowding always damaged their optical-electrical performances. Optimizing the LED chip design through special n-contact electrodes had been reported to improve the current spreading [11-14]. Lv. J. J et al. reported that the LED chip with distributed via-holes exhibited great impact on the current spreading and the subsequent optoelectronic characteristics. Based on the thermal and parasitic resistance analysis, Yin L.Q. et al. indicated that better performances of LED with through-via structure was attributed to the lower chip thermal resistance (i.e. lower heat-flux generation) and lower extra parasitic series resistance (i.e. lower voltage parameters) compared with conventional LED chips. The series resistance was caused by the current-spreading structure and the gold wire connection. In ref [15], a current-confined aperture was proved to broaden the $-3 \mathrm{~dB}$ modulation bandwidth of a micro-LED chip effectively.

In this paper, we intend to increase both the optoelectronic performances and $-3 \mathrm{~dB}$ modulation bandwidth of a medium power GaN-based blue LEDs through periodic via-holes. Experimentally, the luminous efficiency and $-3 \mathrm{~dB}$ modulation bandwidth of the LEDs with periodic via-holes are increased by ratios of $33 \%$ and $48 \%$, respectively, compared with the control LEDs without periodic via-holes.

\section{Experiments}

Commercially available GaN-based LED epitaxy wafers are used here. The GaN-based epi-stacks are grown on the c-plane patterned sapphire substrate and consist of a 30nm-thick GaN nucleation layer, a $1.5 \mu \mathrm{m}$-thick undoped GaN layer, and a $5 \mu \mathrm{m}$-thick Si-doped $\mathrm{n}$-GaN layer, an active region with five periods of InGaN/GaN multi-quantum wells (MQWs), 50nm-thick Mg-doped AlGaN and a 101nm thick Mg-doped p-GaN layer.

The device fabrication begins at immersing the wafer into boiling gaquaregia $\left(\mathrm{HCl}: \mathrm{HNO}_{3}=3: 1\right)$ for 10 minutes, and rinsing it in running deionized (DI)water. Prior to lithography, the wafer is ultrasonically degreased by acetone, methanol, and DI 
water for 5 minutes at each step, followed by $\mathrm{N}_{2}$ blowing. After the cleaning process, mesa structures are defined by inductively coupled plasma (ICP) etching. The ICP is also used to etch away the p-GaN, MQWs, and part of n-GaN to form periodic via-holes. The $\mathrm{Ni} / \mathrm{Au}(5 \mathrm{~nm} / 7 \mathrm{~nm})$ conductive layers are evaporated on the $\mathrm{p}-\mathrm{GaN}$ surface by electron beam and are treated to form ohmic contacts by rapid thermal annealing (RTA) at $550^{\circ} \mathrm{C}$ for $15 \mathrm{~min}$ in $\mathrm{N}_{2}$ ambient. Then, all surfaces of the chip are passivated by $\mathrm{SiO}_{2}$ through plasma-enhanced chemical vapor deposition (PECVD). After removing the parts of the $\mathrm{SiO}_{2}$ layer to expose the bottom of the via-holes and the p-ohmic contact, $\mathrm{Cr} / \mathrm{Pd} / \mathrm{Au}(20 / 40 / 200 \mathrm{~nm})$ multilayers are deposited to form n-pad and p-pad. Finally, the sapphire substrate is lapped and polished down to $80 \mu \mathrm{m}$; LED chips are cut into separate pieces and packaged by standard technique. The active area of each chip is $200 \mu \mathrm{m} \times 800 \mu \mathrm{m}$ and the diameter of the via-hole is designed as $50 \mu \mathrm{m}$. For comparison, an LED chip with only one big via-hole $($ Diameter $=86.6 \mu \mathrm{m})$, which has the same active area as the fabricated sample LEDs with periodic via-holes, is also fabricated under the same conditions, denoted as "the control LED" in the following. In other words, these two kinds of LED chips have an equal effective PN junction area, as shown in Fig.1 [16]. The fabricated LED chips are bonded onto the leadframe packages and encapsulated by standard procedures. The optical microscope images of all LEDs fabricated in this paper are shown in Fig.2. In order to exclude the effect of lower extra parasitic parameters on the improvement of $-3 \mathrm{~dB}$ bandwidth, wire bonding is used for electrode connections.

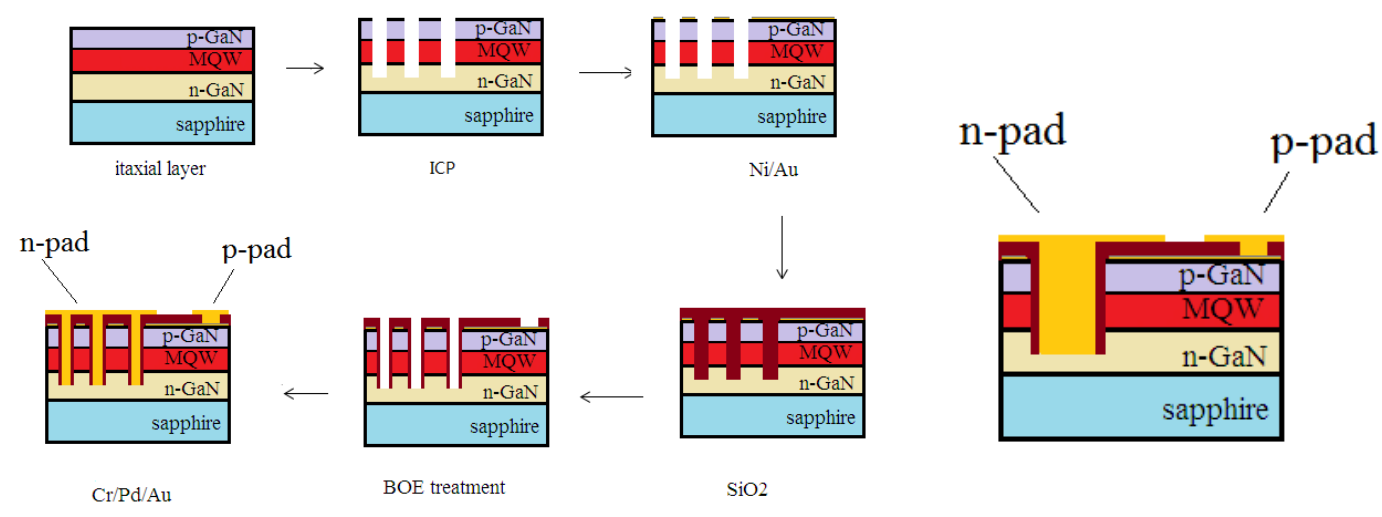


Fig.1. Fabrication processes for the two kinds of LED chips: (a) the LED chip with periodic micro via-holes, denoted as "the sample LED"; (b) the LED chip without periodic via-holes, denoted as "the control LED".

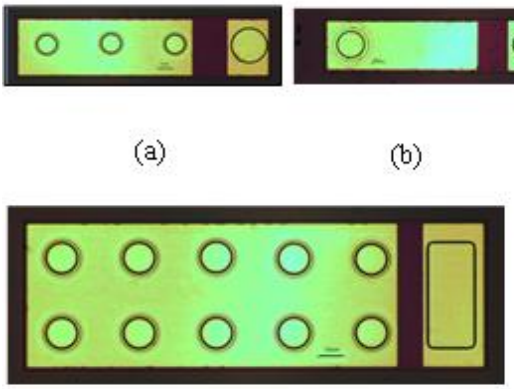

(e)

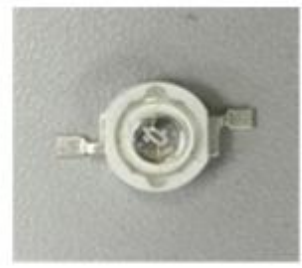

(g)



(c)



(f)

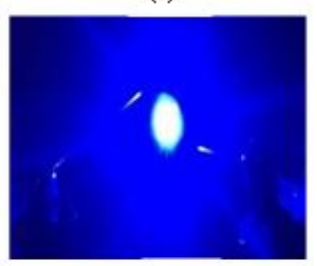

(h)

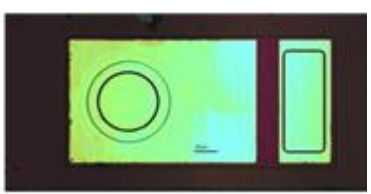

(d)

Fig.2. Optical microscope images of LEDs: (a)LED with 3 periodic micro via-holes and (b)its control sample: LED without periodic micro via-holes(i.e. only one big via-hole); (c) LED with 6 periodic micro via-holes and (d)its control sample: LED without periodic micro via-holes; (e) LED with 10 periodic micro via-holes and (f)its control sample: LED without periodic micro via-holes. (g) The packaged LED. (h)

The LED chip with 6 periodic micro via-holes operating at $\mathrm{I}=10 \mathrm{~mA}$.

The electroluminescence (EL) spectra and current-voltage characteristics are measured by Agilent B1500A Semiconductor Parameter Analyzer. The output power of the LEDs is measured by integrated sphere (HAAS 2000). The capacitance-voltage measurement is carried out on Agilent B1505A. The frequency responses of two LEDs are characterized by a network analyzer (Agilent 5071C) and the block diagram of the measurement setup is shown in Fig.3.

The $-3 \mathrm{~dB}$ modulation bandwidth measurement system is comprised of a network analyzer (Agilent 5071C), an amplifier, a bias-tree (mini-circuits ZX85-12G+), a DC 
supply, an LED under test, and a PIN photo-detector (New port 818-BB-21A). The network analyzer provides a small-signal sine wave that is amplified to increase the LED modulation depth. The amplified signal is then coupled with the bias direct current through a bias-tee. Lights from the LED are transmitted through free space and detected by the photo-detector. Finally, the output electric signal of the photo-detector is sent to the network analyzer for generating electrical-to-optical (E-O) response curves, from which the $-3 \mathrm{~dB}$ modulation bandwidths can be evaluated.

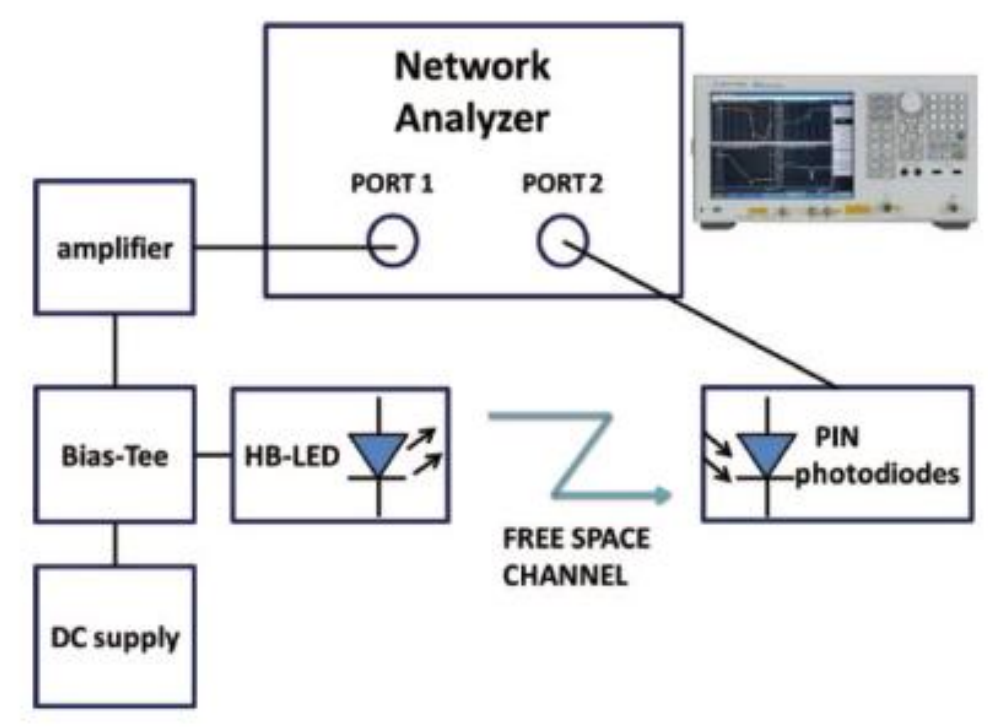

Fig.3. Block diagram of the setup for measuring the frequency responses of LEDs.

\section{Results and discussions}

Electrical and optical performances of the blue LEDs with periodic via-holes and without periodic via-holes are characterized. The two kinds of LED chips are designed to have an equal effective PN junction area. Their EL spectra are compared in Fig. 4. Obviously, the LED with periodic via-holes exhibits a $4 \mathrm{~nm}$ red shift $(450 \mathrm{~nm}$ to $454 \mathrm{~nm}$ ), a larger full-width-at-half-maximum (FWHM) and a higher light intensity compared with the control LED. The reason shall lie in the different stress states in these two kinds of LEDs. One big hole in the LED might release more growth stresses inside the epitaxy layers than periodic small via-holes, thus the blue shift may partly 
be resulted from the quantum-confined stark effect (QCSE) $[17,18]$. But the periodic small via-holes will generate more uniform stress and current distributions in the active region than only one big hole, it is expected that the internal quantum efficiency of the LED can be improved by periodic small via-holes [11-14]. In addition, when the



wider FWHM than the control LED. A larger perimeter also means a larger sidewall area, which will benefit light extraction efficiency.

Fig.4. Electroluminescence (EL) spectra of LEDs with and without periodic micro via-holes.

Fig.5 show the I-V characteristics of the two kinds of LEDs. The turn-on voltages of the LEDs both are around 2.7V. The series resistances, which are extracted from 
the above I-V curves, were about $22.98 \Omega$ and $23.8 \Omega$, respectively. Since the series resistances have relations with current spreading, the smaller series resistance proves that the sample LED with periodic via-holes has better current spreading uniformity over the active region $[11,19]$.

The optical powers of LEDs with periodic via-holes and the control LED are measured and plotted as a function of the driving current from $50 \mathrm{~mA}$ to $350 \mathrm{~mA}$, as shown in Fig.6. The LED with periodic via-holes not only exhibits higher optical powers at the same input current, but also has a larger "roll over" current value. These can safely lead to the conclusions that the periodic via-holes lead to the better uniformity of current spreading, better heat dissipation and improved the light extraction efficiency [19-21].

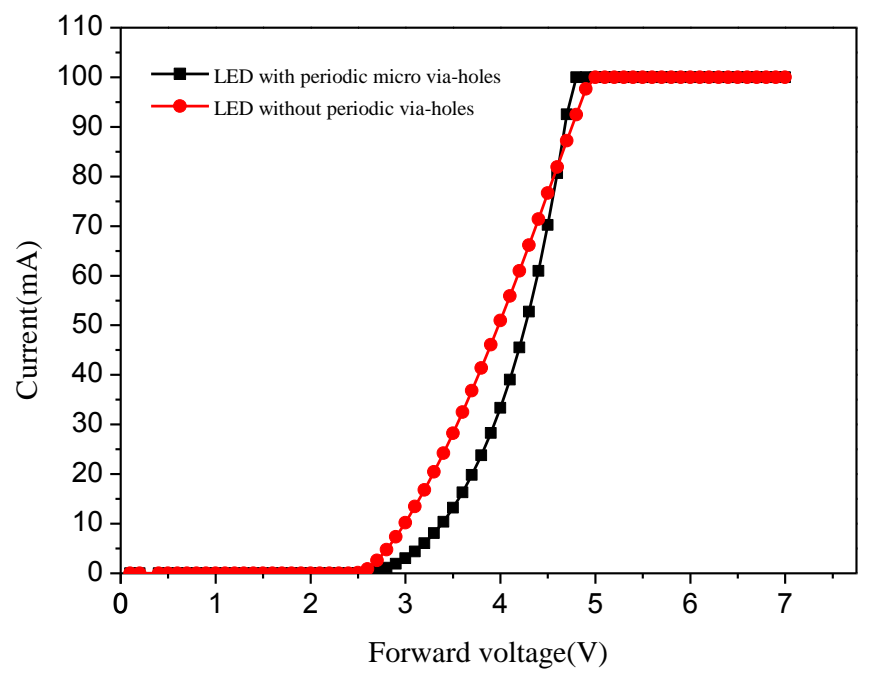

Fig.5. The I-V characteristics of the LEDs with and without periodic via-holes. 


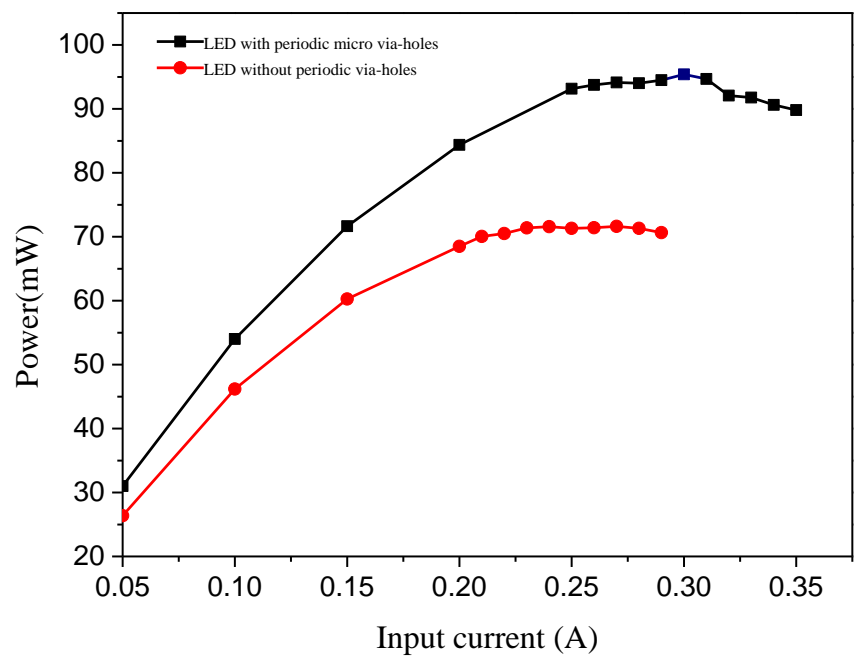

Fig.6. Curves showing the output power as a function of injection current for the two kinds of LEDs. 
Fig.7 plot the normalized frequency responses of two kinds of LEDs at different currents. It can be seen that as the applied current increases, the $-3 \mathrm{~dB}$ modulation bandwidth also increases. The phenomenon was thought to be caused by the possible lower carrier lifetime at high current densities [7, 22]. Fig.8 further clarifies the dependence of the $-3 \mathrm{~dB}$ bandwidth on the injected current. As the forward current increases, the LED with periodic via-holes always presents a larger $-3 \mathrm{~dB}$ bandwidth.
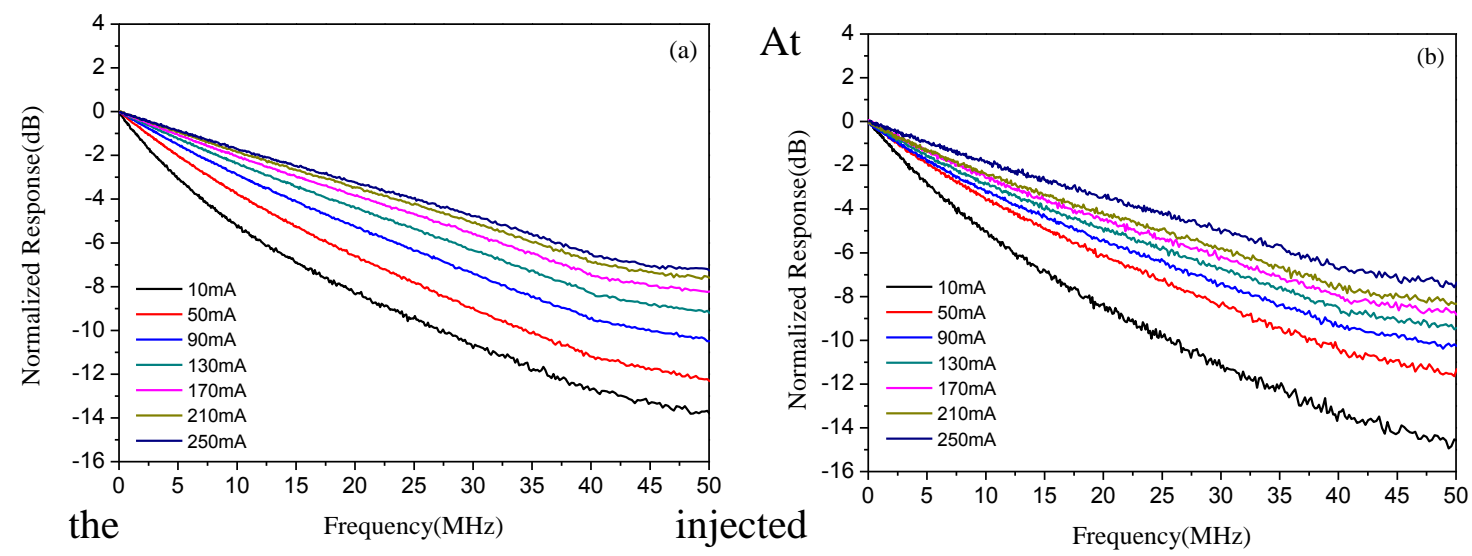

current of $250 \mathrm{~mA}$, the $-3 \mathrm{~dB}$ bandwidths of the two LEDs with and without periodic via-holes are $18.4 \mathrm{MHz}$ and $12.5 \mathrm{MHz}$, respectively. In order to verify this result, we design additional two groups (group I and group II) of medium power LED chips. The group I has a PN junction area $300 \mu \mathrm{m} \times 600 \mu \mathrm{m}$ and 6 periodic via-holes. The group II has a PN junction area $300 \mu \mathrm{m} \times 900 \mu \mathrm{m}$ and 10 periodic via-holes. The diameter of each hole is fixed at $50 \mu \mathrm{m}$.

Fig.7. The normalized frequency responses of LEDs at different currents: (a) LED with periodic micro via-holes, (b) LED without periodic via-holes.

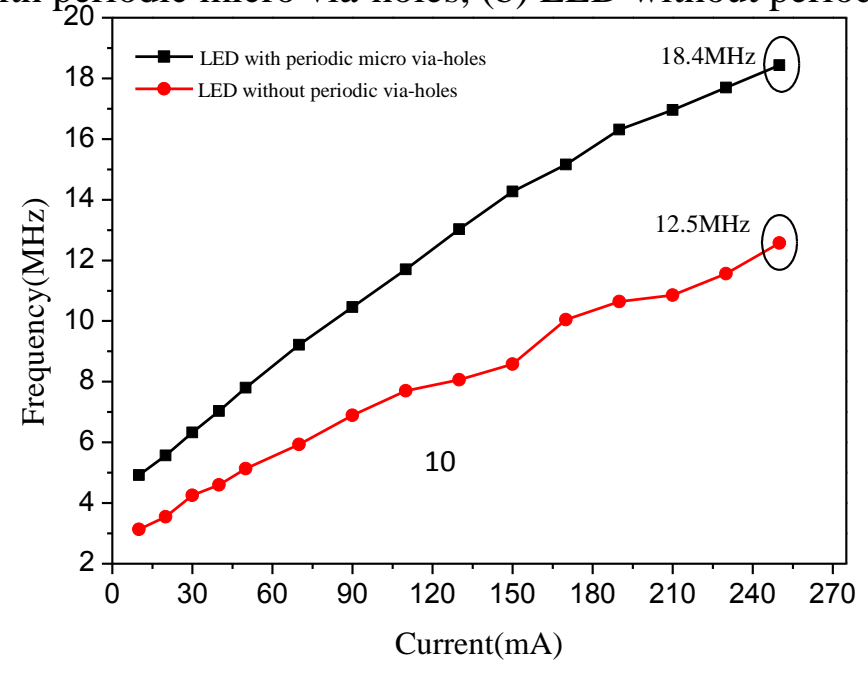


Fig.8. The dependence of the $-3 \mathrm{~dB}$ bandwidth on the injected current for LEDs with and without periodic micro via-holes.

The dependences of the $-3 \mathrm{~dB}$ bandwidths on the injected currents for these two groups of LEDs are plotted in Fig.9. Again, the LEDs with periodic via-holes exhibit higher $-3 \mathrm{~dB}$ modulation bandwidths than their control partners for the two groups. These results demonstrated that the periodic via-holes can improve $-3 \mathrm{~dB}$ modulation bandwidth of LEDs effectively.
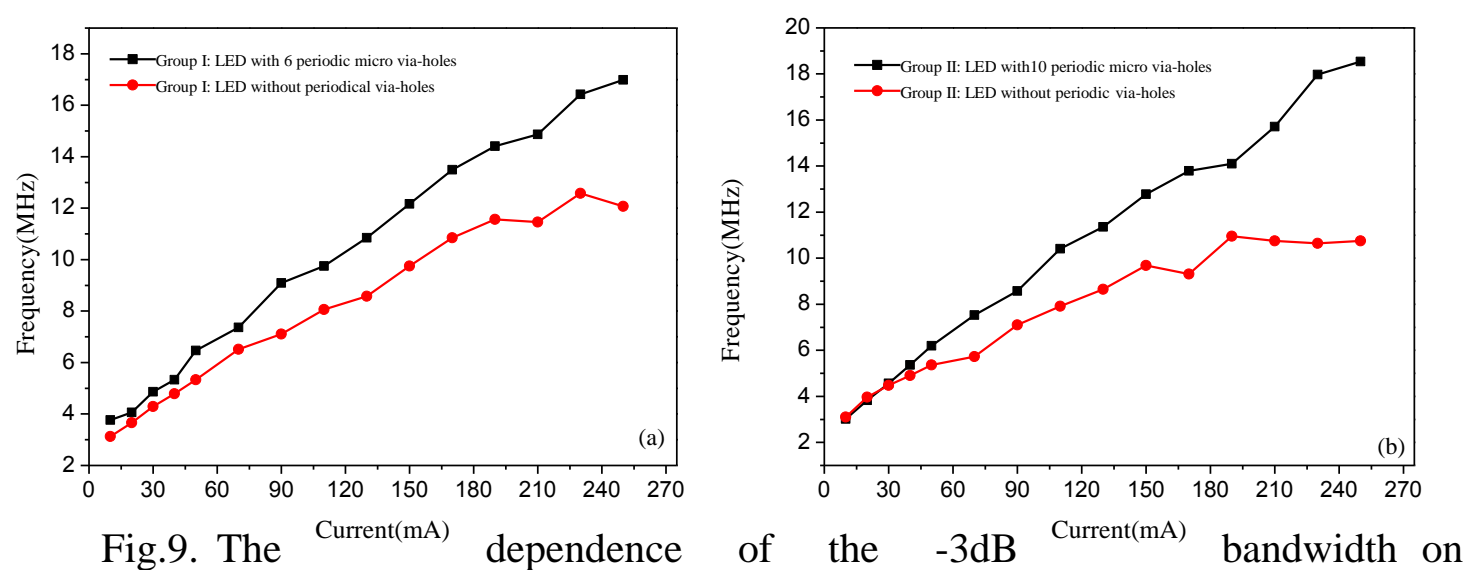

the injected current for additional two groups of LEDs with and without periodic via-holes: (a) Group I. (b) Group II.

There are two major factors limiting the modulation bandwidth, one is the minority carrier lifetime and the other is RC time constant [23-27]. The two factors have different effects on the modulation bandwidth under different current densities. The RC delay time seems to be a limiting factor only at the low current density 
$\left(<1.6 \mathrm{KA} / \mathrm{cm}^{2}\right)$ and the carrier lifetime plays the role at the higher current density $\left(>1.6 \mathrm{KA} / \mathrm{cm}^{2}\right)$ [28]. In present experiments, the fabricated medium power LED chips work under relatively low bias current density $\left(<1.6 \mathrm{KA} / \mathrm{cm}^{2}\right)$, the RC time constant shall play the major role on $-3 \mathrm{~dB}$ modulation bandwidth. Thus, the $-3 \mathrm{~dB}$ modulation bandwidth can be estimated by [29]:

$$
\mathrm{f}=\frac{1}{2 \pi R C}
$$

Where $\mathrm{R}$ is the series resistance and $\mathrm{C}$ is the diode capacitance.

The capacitances of the two LEDs are measured as a function of the applied voltage, as drawn in Fig.10. The frequency of the input electrical signals is set as $1 \mathrm{MHz}$ during the measurement. Combing the measurement results shown in Fig. 5 and Fig. 10, values of $\mathrm{R}$ and $\mathrm{C}$ of LEDs can be extracted. The sample LED with periodic via-holes has a smaller $\mathrm{R}$ value and a larger $\mathrm{C}$ value than the control LED, i.e. $22.98 \Omega$ versus $23.8 \Omega$, and $403 \mathrm{pF}$ versus $327 \mathrm{pF}$. Based on $\mathrm{R}$ and $\mathrm{C}$ values, the estimated $-3 \mathrm{~dB}$ bandwidth values of LEDs with and without periodic via-holes are $17 \mathrm{MHz}$ and $19 \mathrm{MHz}$, respectively. Obviously, the estimated $-3 \mathrm{~dB}$ bandwidth values of LEDs with and without periodic via-holes are different from those measured. A possible explanation is $\mathrm{R}$ and $\mathrm{C}$ values may vary with the frequency of applied electrical signals. Another explanation is related with the periodic via-holes, which work just like micro-cavities. The "cavity effect" will increase the current densities of regions around the via-holes, which promotes carriers' recombination rates locally. In other words, the periodic via-holes increase the $-3 \mathrm{~dB}$ modulation bandwidth through reducing the carrier lifetime [30]. Undoubtedly, the periodic micro-via-holes in LEDs can effectively enhance their $-3 \mathrm{~dB}$ modulation bandwidth and luminous efficiency. 




Fig.10. The C-V characteristics of two LEDs with and without periodic via-holes

$$
(\mathrm{f}=1 \mathrm{MHz})
$$

\section{Conclusions}

In summary, through fabricating periodic micro via-holes in LED chip, the medium power LED chip exhibits higher output optical power and the $-3 \mathrm{~dB}$ modulation bandwidth by about $33 \%$ and $48 \%$, respectively, compared with the LED without periodic micro via-holes. The mechanism shall be due to that periodic micro via-holes improve the current spreading, heat dissipation and carriers' recombination rates. The method of concurrently improving modulation and optical performances of power-type LED chips through periodic micro via-holes take the advantages of easy fabrication, suitable for mass-production. The power-type LED with periodic micro via-holes pave a way for the application in illumination and high speed communication dual-usage VLC system.

\section{Acknowledgments}

The authors acknowledge support by the grants from Natural National Science Foundation of China (NSFC) (U1201254); Program 863 (2015AA03A101); Guangzhou Technical Plan (201510010280); Guangdong Technical Plan (2014B010119003， 2014B010122005， 2015B010113002， 2016B010111002); 
Natural Science Foundation of Guangdong (2016A030313336, 2015A030312011).

\section{References}

[1] D. Tsonev, H. Chun, S. Rajbhandari, J. J. D. McKendry, S. Videv, E. Gu, M. Haji, S. Watson, A. Kelly, G. Faulkner, M. Dawson, H. Haas, and D. O'Brien, IEEE Photon. Technol. Lett., 26 (2014) 637.

[2] H. Le-Minh, D. C. O’Brien, G. Faulkner, L. Zeng, K. Lee, K. Lee, D. Jung, and Y. Oh, IEEE Photon. Technol. Lett. 20, (2008)1243.

[3] H. Li, X. Chen, J. Guo, and H. Chen, Opt. Express 22, (2014)27203.

[4] H. Le-Minh, D. C. O’Brien, G. Faulkner, L. Zeng, K. Lee, K. Lee, D. Jung, Y. Oh, and E. T. Won, IEEE Photon. Technol. Lett. 21(2009)1063.

[5] H. Li, X. Chen, B. Huang, D. Tang, and H. Chen, IEEE Photon. Technol. Lett. 26 (2014) 119.

[6] H. L. Li, Y. N. Zhang, X. B. Chen, C. H. Wu, J. Q. Guo, Z. Y. Gao, H. D. Chen, CHINESE OPTICS LETTERS, 113 (2015) 080605.

[7] P. Tian, J. J. D. McKendry, Z. Gong, S. Zhang, S. Watson, D. Zhu, et al., Journal of Appl. Phys., 115(2014) 033112-1.

[8] D. H. Kwon, S. H. Yang, S. K. Han. Proc. of SPIE, 9387 (2015) 93870T-1.

[9] C. H. Yeh, C. W. Chow, Y. L. Liu et al., Opt. Quant. Electron., 47 (2015) 1991.

[10] C. S Gong, Y. C Lee, J. L. Lai, C. H. Yu, L. R Huang, C. Y. Yang, Scientific Reports. 6 (2016) 30991.

[11] D. A. Zakheim G. V. Itkinson, M. V. Kukushkin, L. K. Markov, O. V. Osipov, A. S. Pavluchenko, I. P. Smirnova1, D. A. Bauman. Phys. Status Solidi. C, 12 (2015) 381.

[12] Y. F. Cai, X. B. Zou, W. C. Chong, K. M. Lau, Phys. Status Solidi A 213 (2016) 1199.

[13] L.Q. Yin, Y. Bai, T.T. Nan, J. H. Zhang, Phys. Status Solidi A, 212 (2015)1725.

[14] J. J. Lv, C. J. Zheng, Q. Chen, S. J. Zhou, S. Liu, Phys. Status Solidi A, (2016) 1.

[15] J. W. Shi, J. K. Sheu, C. H. Chen, G. R. Lin, and W. C. Lai. IEEE Elctron Device 
Letters, 29(2008)158.

[16] S. J. Zhou, S. Yuan, Y. C. Liu, L. J. Guo, S. Liu, H. Ding, Appl. Surf. Sci., $355(2015) 1013$.

[17] S. X. Zhu, J. X. Wang, J. C. Yan, Y. Zhang, Y. R. Pei, Z. Si, H. Yang, L. X. Zhao, Z. Liu, J. M. Li, ECS Solid State Letters, 3 (2014) R11.

[18] S. H. Park and S. L. Chuang, Appl. Phys. Lett., 72, 3103 (1998).

[19] H.M. Jung,G.Y. Nam.,B.K. Choi, T.H. Lee,H.S.Km, S.K. Jeon, E.H. Parkand.T. Kim, Appl. Phys. Lett., 91(2007)1111061.

[20] D. S. Meyaard, Q. F. Shan, J. Cho, E. F. Schubert, S.H. Han, M.H. Kim, C. Sone, S Jae Oh, J. K. Kim, Appl. Phys. Lett.,100(2012)0811061.

[21] J.Han, D. Lee,B. Jin,H. Jeong, J. SongT.Y. Seong, Materials Science in Semiconductor Processing, 31(2015)153. 